\title{
Editorial
}

\section{Toxoplasma gondii centennial anniversary: 100 years of research to celebrate all over the world}

In May 2006, researchers participating in the International Toxoplasma and Food Congress, hosted by the Instituto Zooprofilattico Sperimentale della Sicilia in the city of Palermo, Italy, expressed their support for an international congress to celebrate the 100th anniversary of the discovery of the parasite Toxoplasma gondii in Brazil. The idea rapidly gained the worldwide support of prominent scientists working in the field. The Congress, entitled "Toxoplasma Centennial Congress: From Discovery to Public Health Management" was held in Brazil last year.

The quality and diversity of studies presented in this meeting prompted us to organise the information presented at the meeting, with the aim of bringing together original articles and reviews that will be important for future advances in this field. We consider this endeavour a success. The present issue of Memórias do Instituto Oswaldo Cruz is a collection of 37 articles covering a wide array of research, ranging from basic cell biology, immunology, proteomics and genome projects to studies on clinical management, public health control through screening programmes, diagnosis and environmental control. It also presents two historical reviews. The first, entitled "Toxoplasma gondii: 1908-2008, homage to Nicolle, Manceaux and Splendore”, was written by David Ferguson. The author describes historical advances in understanding the basic biology of the parasite. The second, "Brazilian contribution for a better knowledge on the biology of Toxoplasma gondii", was written by Wanderley de Souza and describes the importance of the works of Herta Meyer, who established the first reproducible studies on the cultivation of T. gondii (Guimarães \& Meyer 1942) and in the 1950s was among the first to use transmission electron microscopy to analyze the fine structure of $T$. gondii.

The original works of Splendore, published in 1908 and 1909, and of Nicolle and Manceaux, published in 1909, are reprinted with permission in the supplementary electronic version of this issue in their respective original languages, Italian and French. An English translation is also available. We invite you to explore these works, which will certainly please and surprise you when you discover the richly detailed descriptions of the parasite that were made using microscopes with inconceivable resolutions for that day's technology. The skill of those scientists was impressive for proposing such accurate hypotheses on the mode of infection and by the challenges that loomed on the horizon for future researchers who dedicated and still dedicate their lives to investigating the many aspects of $T$. gondii and the relationship with its hosts.

The challenges of understanding $T$. gondii still arise today and will certainly pave the way for scientific research throughout this century. Splendore and Nicolle exchanged slides with preparations from infected rabbits and gundis, respectively, and were convinced that the new protozoa belonged to the same genus. They independently described the parasite dividing by "longitudinal bipartition" (currently known as endodyogeny). This form of asexual multiplication is illustrated in the electron micrograph used on the cover of this issue. Splendore also suggested schizogony as a form of division, but Nicolle believed that it probably represented repeated cycles of "longitudinal bipartition" (repeated endodyogeny). However, a specific form of schizogony (Toxoplasma endopolygeny) occurring during coccidian development in the gut of the cat was identified 60 years later.

In the second publication on the subject (1909), Splendore describes three very important aspects of the biology of Toxoplasma cuniculi (as he named the new parasite on that occasion) and the signs of the disease it caused: (i) "In a new group of cases occurring in the same period last year, microscopic examination revealed the same anatomic and parasitic situation. I am of the opinion that seasonal variation must be taken into consideration in a transmission study, as constant transmission has never been possible." (ii) "A dog injected with emulsified tissues extracted from a rabbit showing a high number of parasites suffered from bloody diarrhoea in the first few days, and two months later was suffering from severe progressive wasting disease and loss of sight due to ocular turbidity." (iii) "Surprisingly, rabbit parasites can reproduce in birds, as shown last year with two sparrows (Zonotrichia pileata) that died five days after subcutaneous injection of two spleen drops extracted from an infected rabbit."

Those three aspects mentioned by Splendore were shown to have the following important public health impacts several years later: (i) seasonal weather variations, for instance, have been considered an important factor in toxoplasmosis outbreaks where oocysts are implicated as the life cycle stage responsible for infection dissemination (Bowie et al. 1997, DeMoura et al. 2006); (ii) the ocular involvement of toxoplasmosis, which was definitively recognised in the late 1940s and early 1950s (Wilder 1952, Feldman 1953, Frenkel \& Jacobs 1958 and Ferguson, in this issue); and (iii) the diversity of $T$. gondii intermediate hosts; namely, the fact that any warm-blooded animal - bird or mammal - is a potential intermediate host (Dubey \& Beatie 1988) was highlighted by Splendore when he noted that the new disease of rabbits could be reproduced in birds.

The concomitant discovery of the parasite in South America and North Africa raised the question: is it exactly the same parasite? One of the major findings of the past 25 years concerns the genetic diversity of $T$. gondii and the geographical distribution of genotypes, South American genotypes being different from European or North American ones. Other continents are still relatively unexplored. 
Substantial advancements in Toxoplasma molecular and cell biology research have also occurred in the past 20 years. This issue includes three critical reviews and viewpoints on specific topics in those areas written by distinguished senior and young scientists who propose ideas that certainly will stimulate new directions for research for the next 100 years. In his article, Boothroyd explores the hypothesis that a combination of sexual recombination and host range expansion may be the major driving force in the evolution of some gene families and the specific genes they encompass. The author proposes central questions that remain to be investigated, such as: "Might some strains of Toxoplasma be adapted to sexual replication in non-feline hosts? How many strains have actually been tested for their ability to infect non-felines?" He concludes, stating: "Perhaps it is time to revisit the 'felines-only' dogma". Sullivan et al. discuss the evidence that shows how the clinically relevant process of bradyzoite differentiation is regulated at both transcriptional and post-transcriptional levels and pose some questions for future research. In the article entitled "What new cell biology findings could bring to therapeutics: is it time for a phenome project in Toxoplasma gondii?" Meissner and Klaus argue that in the absence of a powerful high-throughput technology for the characterization of essential genes of interests, a coordinated effort should be undertaken to convert the knowledge of the genome into that of the phenome.

The impact of the development on Toxoplasma genetics research can be evaluated in the articles presented in this issue by Jamielson et al., who tested the hypothesis that propensity for $T$. gondii to cause eye disease is associated with genes previously implicated in congenital or juvenile onset ocular disease, and by Dubey and Su, who compared Toxoplasma strains from United States and Brazil and concluded that different population structures of T. gondii between these countries may be shaped by different transmission routes. On the topics of molecular cell biology research and the host-parasite relationship, the articles published by Guimarães and Ferreira-da-Silva provide new data on the potential use of primary cultures of skeletal muscle cells as a cellular model for in vitro differentiation of the RH strain. Milovanović et al. present a set of data showing that significant lipid metabolism alterations, including a decrease in reverse cholesterol transport, are observed during acute Toxoplasma infection.

Substantial advances have also been achieved over the past 15 years in basic and applied research on the host immune response against Toxoplasma. Nine articles on this subject are published in the present issue. Five excellent reviews are presented. Hunter and Tait explore the development of a working model to study immunity to T. gondii, Garweg and Candolf discuss current facts and possible clues on the immunopathology of ocular toxoplasmosis and Subauste proposes that cell-mediated immunity through CD40 reroutes the parasitophorous vacuoles (PVs) to the lysosomal compartment by a process called autophagy, resulting in the death of $T$. gondii and thus challenging the dogma that the non-fusogenic nature of the PV is irreversible. Zaho et al. present a review on the immunity-related GTPase resistance system in mice. Schreiner and Liesenfeld present a review entitled "Small intestinal inflammation following oral infection with Toxoplasma gondii does not occur exclusively in C57BL/6 mice: review of 70 reports from the literature", in which the authors review the common features of small intestinal immunopathology in the animal kingdom and speculate on its consequences for immunopathology in humans. Two articles present data on the immune response of hosts. Brandão et al. report the immune response of experimental reinfection of BALB/c mice to different recombinant type I/III strains of T. gondii, and Bahia-Oliveira et al. describe the evidence for the role of cross-immunomodulation in the outcome of ocular toxoplasmosis in areas endemic for T. gondii and Ascaris lumbricoides. In the area of vaccinology, two reviews are presented. Innes et al. report some of the history, challenges and progress in the development of veterinary vaccines against $T$. gondii, Jongert et al. propose that future studies should use animal models other than rodents and that goals such as the prevention of maternal-foetal transmission and eye disease are as important as the traditional goals of survival or reduction in brain cyst numbers.

Environmental research on toxoplasmosis fortunately has gained even more importance. Three articles are presented on this subject: Villena et al. report methods for the detection of Toxoplasma oocysts in water and their application in the Champagne-Ardenne Region of France, Forman et al. describe the seroprevalence of T. gondii in British marine mammals and Mai et al. present an important review on oocyst wall formation and composition in coccidian parasites, factors that contribute to oocyst resistance to a variety of environmental and chemical insults.

Due to the effectiveness of sulphonamides against murine toxoplasmosis, reported by Sabin and Warren (1942), and the synergistic effect of combined therapy with sulphonamides and pyrimethamine, described by Eyles and Coleman (1953), these drugs form the basis for the standard therapy regimens for toxoplasmosis in humans (Remington et al. 2005). However, more than 50 years after the first assays on drug management of toxoplasmosis, drug efficacy in the prevention of congenital toxoplasmosis and the treatment of ocular disease is still a matter for debate, as reported in five articles in this issue: (Chêne \& Thiébaut, Gilbert, McLeod et al., Stanford \& Gilbert and Peyron). Research on drugs should be one of the major goals of the next century, as emphasized in the presentations of several delegates at the meeting. This is the ultimate goal of cell biology studies, according to Sullivan et al. Development of a vaccine is another approach, both to reducing Toxoplasma burden in animals destined for meat production and to preventing human infection (Innes et al., Jongert et al.).

We can only honour the legacy of the scientists awarded with the first edition of the Splendore and Nicolle Medal (see the foreword of this issue) on the progress of research in parasitology clinical management, diagnostics, immunology and molecular biology of $T$. gondii. Their works have directed efforts not only on the basic biology 
of the parasite but also in clinical management and public health areas of Toxoplasma research worldwide. In the field of public health, there are one review written by Tenter discussing the routes that are most important from a public health point of view, considering the transmission of the parasite to humans. It is also presented four articles in this issue with data from The Netherlands (Kortbeek et al.), Italy (Stagni et al.) and Brazil, where infection is a major health problem (Lopes et al., Varella et al.). On the topic of diagnosis, articles are presented by Bessières et al., on congenital toxoplasmosis in France, by Neves et al., on acute acquired toxoplasmosis, and by Mesquita et al., on cerebral toxoplasmosis in peripheral blood samples from HIV-infected patients in Brazil. G Holland presents an article on clinical management of ocular disease entitled "Ocular toxoplasmosis: the influence of patient age", in which the author discusses the influence of patient age on ocular toxoplasmosis, concluding that it should be studied systematically in order to provide both a better understanding of disease mechanisms and clinical information that may be used to establish better treatment and prevention strategies. Comodaro et al. present an overview and update on ocular toxoplasmosis, addressing many misconceptions still found in the medical literature and Melamed presents a history of the ocular toxoplasmosis knowledge gained from studies and observations performed in Southern Brazil over the past 30 years.

Sadly, two months prior to the meeting held last September, Dr. George Desmonts, one of scientist who received the Splendore and Nicolle medal, died. However, his contribution to toxoplasmosis research is immortalised in the literature. One example is his and Jacques Couvreur's 1974 publication in the New England Journal of Medicine (Desmonts $\&$ Courvreur 1974). This major publication revealed the importance of maternal-foetal transmission of $T$. gondii and its devastating consequences. This paper still remains a basic reference for anyone working on toxoplasmosis.

In our opinion, however, the major contribution by George Desmonts and his contemporary colleagues was their effort to have congenital toxoplasmosis included in the list of diseases that deserved special attention from the French public health authorities. The inclusion of congenital toxoplasmosis in such a list resulted in the establishment of a special programme to prevent, diagnose and treat congenital infections, an example to be followed by many countries.

The present issue of Memórias do Instituto Oswaldo Cruz is an homage not only to the pioneers Alfonso Splendore, Charles Nicolle and Luis Manceaux, but also to all the researchers and students who dedicated, continue to dedicate and shall dedicate their efforts to understanding the fascinating biology of this parasite and its relationships with hosts. This applies especially to those who have fought relentlessly to include toxoplasmosis, particularly congenital toxoplasmosis, in the list of diseases that are neglected and that deserve special attention from public health authorities worldwide, especially in places with alarming levels of infection. Finally, we extend this homage to toxoplasmosis victims, many of whom will never see or benefit from the success of these research efforts.

\section{Lílian MG Bahia-Oliveira}

Marie-Laure Dardé

Maria Regina Reis Amendoeira

\section{REFERENCES}

Bowie WR, King AS, Werker DH, Isaac-Renton JL, Bell A, Eng SB, Marion SA 1997. Outbreak of toxoplasmosis associated with municipal drinking water. The BC Toxoplasma Investigation Team. Lancet 350: 173-177.

De Moura L, Bahia-Oliveira LMG, Wada MY, Jones JL, Tuboi SH, Carmo EH, Ramalho WM, Camargo NJ, Trevisan R, Graça, RMT, da Silva AJ, Moura I, Dubey JP, Garrett DO 2006. Waterborne outbreak of toxoplasmosis, Brazil, from field to gene. Emerg Infect Dis 12: 326-329.

Desmonts G, Couvreur J 1974. Congenital toxoplasmosis. A prospective study of 378 pregnancies. N Engl J Med 290: 1110-1116.

Dubey JP, Beattie CP 1988. Toxoplasmosis of animals and man, CRC Press, Boca Raton, 220 pp.

Eyles D, Coleman N 1953. Synergistic effect of sulphadiazine and daraprim against experimental toxoplasmosis in the mouse. Antibiot Chemother 3: 483-490.
Feldman HA 1953. The clinical manifestations and laboratory diagnosis of toxoplasmosis. Am J Trop Med Hyg 2: 420-428.

Frenkel JK, Jacobs L 1958. Ocular toxoplasmosis. Arch Ophthal (Chicago): 48: 260-279.

Guimarães FN, Meyer H 1942. Cultivo de "Toxoplasma" Nicolle \& Manceaux, 1909, em cultura de tecidos. Rev Bras Biol 2: 123-129.

Remington JS, McLeod R, Thulliez P, Desmonts G 2006. Toxoplasmosis. In J Remington, G Klein, C Wilson, C Baker, Infectious diseases of the fetus and newborn infant, 6th edition, WB Saunders, Philadelphia, p. 947-1091.

Sabin AB, Warren J 1942. Therapeutic effectiveness of certain sulfonamide on infection by an intracellular protozoon (Toxoplasma). Proc Soc Exp Biol Med 51: 19-23.

Wilder HC 1952. Toxoplasma chorioretinitis in adults. AMA Arch Ophthalmol 48: 127-136. 